\title{
Reduction of Channeling Effects in Aberration-Corrected STEM by Orientating the Sample Along Low Atomic Linear Density Zone Axes.
}

\author{
D. Hernández-Maldonado*, M. Herrera*, M. P. Guerrero-Lebrero**, J. Pizarro**, P. L. \\ Galindo** and S. I. Molina* \\ *Departamento de Ciencia de los Materiales e I.M. y Q.I., Facultad de Ciencias, \\ Universidad de Cádiz, Campus Río San Pedro, s/n, 11510 Puerto Real, Cádiz, Spain. \\ ** Departamento de Lenguajes y Sistemas Informáticos, CASEM, Universidad de Cádiz, \\ Campus Río San Pedro, s/n, 11510 Puerto Real, Cádiz, Spain.
}

Developments in aberration-corrected Scanning Transmission Electron Microscopy (STEM) have improved lateral resolution and shortened the depth field, opening the possibility of performing $3 \mathrm{D}$ reconstructions of nanoscale objects from the analysis of through-focal series [1]. Imaging of individual dopant atoms embedded into amorphous [2] and into crystalline materials [3] by optical sectioning have been demonstrated. In crystalline materials channeling effect along atomic columns alters the shape of the probe, creating artifacts and complicating a quantitative interpretation of STEM images. In order to reduce this effect different approaches have been done [4,5], but until now, none of them has reduced the channeling enough to reconstruct nanoscale objects. Another approach to reduce the channeling is to introduce the probe along a zone axis of low atomic linear density. The idea is to orientate the sample along a zone axis where the distance between atoms are big enough like to make difficult the propagation of the probe through the sample.

The aim of this work is to analyze the variation of the channeling when the probe is introduced into the sample along low atomic linear density crystallographic orientations. Simulations have been carried out with SICTEM [6], an HAADF image simulation software, which is able to simulate nanostructures, developed for running on the CAI University of Cadiz Supercomputer, for a $300 \mathrm{kV}$ dedicated VG603 STEM $\left(\mathrm{C}_{\mathrm{s}}=-0.037\right.$ $\mathrm{mm}, \mathrm{C} 5=100 \mathrm{~mm}$, inner detector angle $=58 \mathrm{mrad}$, outer detector angle $=200 \mathrm{mrad}$ and objective aperture $=22 \mathrm{mrad}$ ).

Figure 1 compares the channeling along two crystallographic orientations of InAs, with the probe focused on an As atom at the surface (defocus=0). It is possible to observe that along the [110] direction, which possesses an atomic linear density of 3.1 atoms $/ \mathrm{nm}$, the channeling is significant bigger $(\sim 15 \mathrm{~nm})$ than along [210] $(\sim 10 \mathrm{~nm})$, which atomic linear density is 1.1 atoms $/ \mathrm{nm}$. When the probe is introduced in the sample along the [210] orientation, distance between atoms is increased with respect the [110], and then channeling effect is reduced. It has been noticed that the channeling is reduced less than $10 \mathrm{~nm}$ along [n10] (when $\mathrm{n} \neq 1$ ) crystallographic orientations. These results are promising and open the possibility to reduce the channeling with the aim to do 3D reconstructions of nanoobjects. Another interesting case where we have explored the channeling is along the strained planes of quantum dots. A reduction of the channeling effect also occurs for a 
focused probe at the surface of a quantum dots when it is compared with an unstrained material.

References

[1] A. Y. Borisevich et al. J. Electron Microsc, 55 (2006) 7

[2] Huolin L. Xin et al. Microsc, Microanal, 16 (2010) 445

[3] S. H. Oh et al., Nano Letters, 8 (2008) 1016

[4] Huoling L. Xin et al. Microsc, Microanal, 14 (Suppl 2) (2008) 926

[5] Huoling L. Xin et al. Appl. Phys. Lett, 92 (2008) 013125

[6] J. Pizarro at al. Appl. Phys. Lett. 93 (2008) 153107

[7] This work was supported by the Spanish MICINN (projects TEC2008-06756C03- and CONSOLIDER INGENIO 2010 CSD2009-00013) and the Junta de Andalucía (PAI research groups TEP-120 and TIC-145; project P08-TEP-03516).

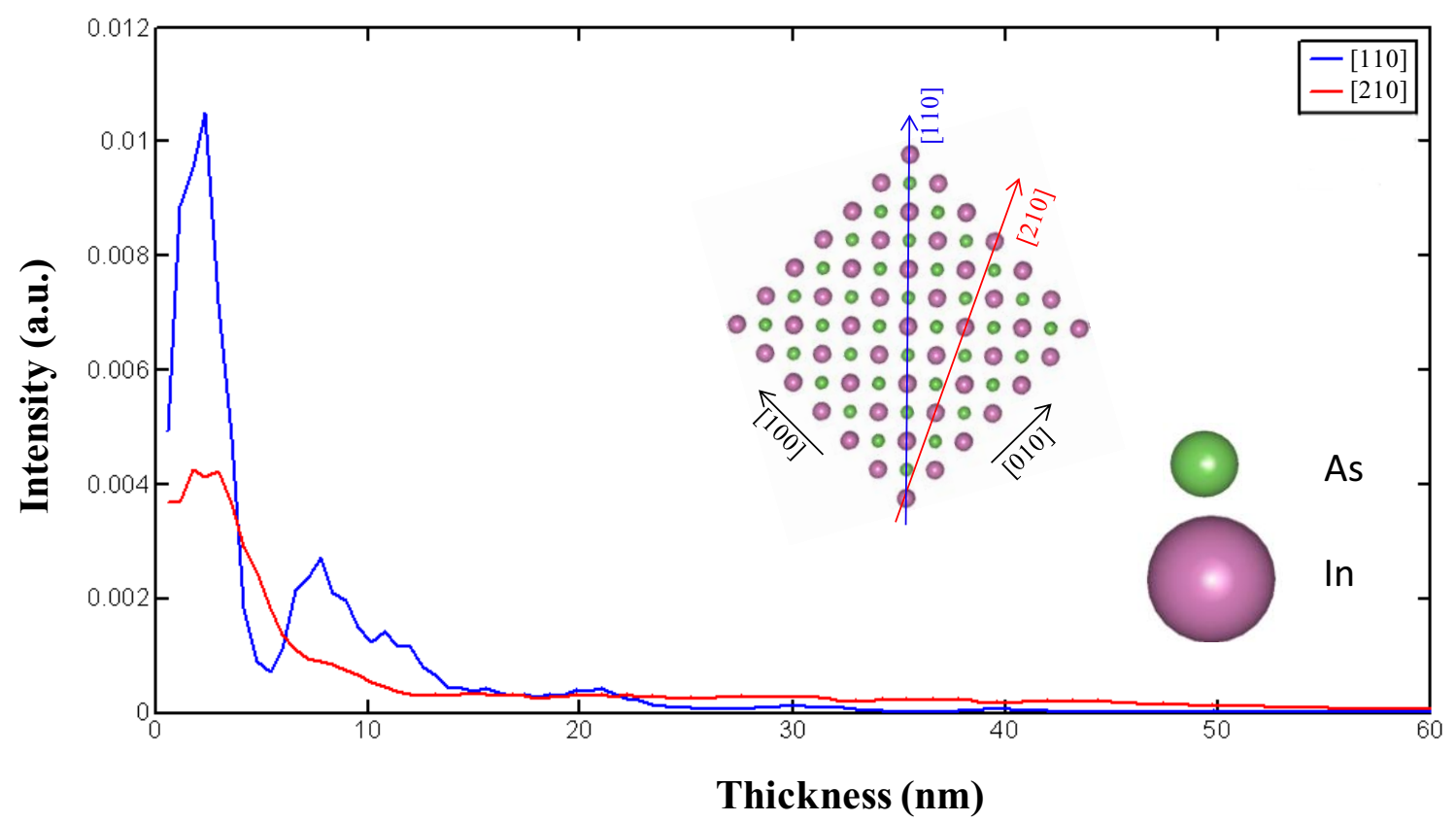

FIG.1 Comparison between the channeling along the [110] and [210] crystallographic orientations in a 60nm-thick InAs sample. In the inset it is represented an InAs crystal, and the two directions along the probe has been introduced into the sample. The blue arrow marks the [110] orientation and the red one the [210]. 